\title{
Correction to: Fast Lottery-Based Micropayments for Decentralized Currencies
}

Kexin $\mathrm{Hu}$ and Zhenfeng Zhang

Correction to:

Chapter "Fast Lottery-Based Micropayments

for Decentralized Currencies" in: W. Susilo and G. Yang

(Eds.): Information Security and Privacy, LNCS 10946, https://doi.org/10.1007/978-3-319-93638-3_38

In the original version of this chapter the second affiliation was missing for both authors. This has now been corrected. The University of Chinese Academy of Sciences has been added as second affiliation. 